\title{
Continuous wound infusion of ropivacaine for the control of pain after thoracolumbar spinal surgery: a randomized clinical trial
}

\author{
Bo $\mathrm{Xu}^{1} \cdot \mathrm{Li} \operatorname{Ren}^{2} \cdot$ Weifeng $\mathrm{Tu}^{1} \cdot \mathrm{Zenghui} \mathrm{Wu}^{3} \cdot \mathrm{Fuzhi} \mathrm{Ai}^{3} \cdot$ Dongxu Zhou ${ }^{1} \cdot$ \\ Biyun Chen ${ }^{1} \cdot$ Xingan Zhang ${ }^{1}$
}

Received: 29 October 2014/Revised: 20 April 2015/Accepted: 20 April 2015/Published online: 3 May 2015

(C) The Author(s) 2015. This article is published with open access at Springerlink.com

\begin{abstract}
Purpose A prospective randomized clinical trial was carried out to observe the analgesic efficacy of ropivacaine for postoperative pain following thoracolumbar spinal surgery.

Methods Seventy-one patients with elective posterior thoracolumbar spinal surgery were randomly divided into two groups. Local group received $0.33 \%$ ropivacaine by pump through the wound, and intravenous group received flurbiprofen axetil, pentazocine and palonosetron via intravenous pump. We evaluated the level of pain, the incidence of adverse reactions at 2, 4, 6, 12, 24, 36 and $48 \mathrm{~h}$ after operation, and the occurrence of chronic pain 3 months later.

Results There were no significant differences in the pain level between the two groups. However, the incidence of nausea, vomiting and chronic pain was significantly lower in the local group.

Conclusions Our results showed that local infusion of ropivacaine achieved similar analgesic effects to
\end{abstract}

B. Xu and L. Ren contributed equally to this work.

RCT Registration Number: NCT01872494.

Bo Xu

xubo333@hotmaill.com

$\triangle$ Weifeng Tu

gzzyyubox@gmail.com

1 Department of Anesthesiology, General Hospital of Guangzhou Military Command, Guangzhou 510010, China

2 Department of Anesthesiology, Zhuhai People's Hospital, Zhuhai 519000, China

3 Department of Orthopedics, General Hospital of Guangzhou Military Command, Guangzhou 510010, China intravenous delivery of analgesic drugs, but significantly reduced incidence of nausea, vomiting and chronic pain.

Keywords Local analgesia - Ropivacaine $\cdot$ Postoperative pain · Chronic pain - Thoracolumbar spinal surgery

\section{Introduction}

Pain is the most commonly reported symptom in primary care after spinal surgery, while musculoskeletal pain accounts for half of all reported pain complaints [1]. The benefits of well-controlled postoperative pain include reduced postoperative cardiopulmonary complications, hospital mortality and length of hospital stay [2]. Modern pain control after major surgery involves a number of different analgesic modalities. Peripheral pain control by incisional local anesthesia is a new method in the treatment of postoperative pain especially after various surgical procedures. Wound infusion with local anesthetics can continuously infiltrate the peripheral nerve endings, and attenuates pain by directly blocking the neuronal membrane sodium channels. A single bolus of local anesthetics in the surgical site can provide a narcotic-limiting pain relief for $4-8 \mathrm{~h}$ after operation [3]. The time-limited effect of single bolus administration can be further improved through continuous surgical wound infusion techniques using multi-orificetype catheters, positioned by the surgeon at the end of the procedure. A systematic review [4] of randomized controlled trials confirmed the benefits and safety of this technique when applied following several severely painful procedures, such as cardiac, thoracic and major gynecologic surgery. This technique remains effective in spinal surgery. Recently, Rushdi et al. [5] reported that the wound was infused with a solution of ropivacaine $0.4 \%$, 
suggesting the potential for the use of this method in major spinal surgery. The current study aimed to test the analgesic efficacy of wound infiltration with $1 \%$ ropivacaine followed by an infusion of $0.33 \%$ following thoracolumbar spinal surgery. Besides, we also evaluated and recorded the long-term effects of incisional local anesthesia on incidence of postoperative chronic pain and infection, compared with postoperative intravenous continuous constantdose analgesia (ICCA).

\section{Materials and methods}

After obtaining approval from the Local Research Ethics Committee, and written informed consent, our study included 71 ASA I-II patients, aged 18-75 years, scheduled for elective thoracolumbar spinal surgery since 2013 May to 2014 February. All 71 patients were randomly assigned to either the local (L) group $(n=35)$ or the intravenous (V) group ( $n=36$ ) using a computer-generated random number table. Exclusion criteria included: a history of cardiopulmonary disease, coagulation and merging with multiple injuries. One anesthesiologist was designated to provide postoperative management.

In the L group, we used continuous wound infusion with ropivacaine as primary analgesia. This group received an initial wound infiltration with $6 \mathrm{ml} 1 \%$ ropivacaine (100 mg; AstraZeneca AB, Sweden) and followed by continuous infusion with $0.33 \%$ ropivacaine via a double lumen catheter system at a rate of $5 \mathrm{ml} / \mathrm{h}$ (Disposable Postoperative Local Analgesia System, Beijing Heng Yuan Tongji Medical Technology Corporation, China) for $48 \mathrm{~h}$. Patient in $\mathrm{L}$ group did not receive postoperative intravenous continuous constant-dose analgesia (ICCA) for pain control. The $\mathrm{V}$ group $(n=36)$ relied on ICCA for postoperative pain control involved flurbiprofen axetil $150 \mathrm{mg}$, pentazocine $240 \mathrm{mg}$ and palonosetron $0.5 \mathrm{mg}$ in $100 \mathrm{ml}$ normal saline, at a rate of $2 \mathrm{ml} / \mathrm{h}$ [5]. All patients expanded on the use of the supplementary analgesic (flurbiprofen $50 \mathrm{mg}$ intravenous injection) if necessary (VAS $\geq 4$ ).

Patients were premedicated with phenobarbital $100 \mathrm{mg}$ and atropine $0.5 \mathrm{mg}, 30 \mathrm{~min}$ before the induction of anesthesia. After baseline measurements of heart rate, noninvasive blood pressure, respiratory rate and oxygen saturation, each patient was preoxygenated for $3 \mathrm{~min}$ before induction. All patients received the target-controlled infusion with propofol 2-3 $\mathrm{g} / \mathrm{ml}$ using the Marsh pharmacokinetic model and remifentanil at 3-4 ng/ml using the Minto pharmacokinetic model for induction. Following the induction of anesthesia, cisatracurium $0.15 \mathrm{mg} / \mathrm{kg}$ was given as an intravenous injection. After tracheal intubation, mechanical ventilation was initiated with $100 \%$ oxygen and adjusted to maintain the endtidal carbon dioxide tension between 35 and $45 \mathrm{mmHg}$. Intermittent bolus injection of cisatracurium was used to maintain full muscle relaxation. At the end of surgery, residual neuromuscular block was reversed, if needed, with a mixture of atropine and neostigmine. Patients were given pentazocine $60 \mathrm{mg}$ when surgery was completed prior to extubation.

\section{Surgery}

In the $\mathrm{L}$ group, after the spinal implant screw and rod system were completed, the surgeon infiltrated all surgical strata and the paraspinal muscles along the wound bilaterally with a solution of ropivacaine $1 \% 6 \mathrm{ml}$. Then, the surgeon inserted two multiholed catheters through an introducer needle, $1-2 \mathrm{~cm}$ from the lower end of the incision along the full length of the wound. The first catheter was positioned close to the vertebral plate and under the paravertebral muscles (deep layer), which were immobilized at the skin. The second catheter was positioned above the deep fascia (superficial layer), and then it was fixed at the skin. A suction drain was placed near the spinal instrumentation level, under the deep fascia and far away from the indwelling catheter. The double catheters were immediately connected to a bacterial filter through which an elastomeric infusion pump delivered $0.33 \%$ ropivacaine at a rate of $5 \mathrm{ml} / \mathrm{h}$ continually until the pump was empty. The analgesia infusion pump was commenced following surgery. Recovery from anesthesia and pain management after surgery were identical for all patients.

\section{Outcome measures}

Demographic and operation data were collected including disease, date of birth, gender, operating time, preoperative VAS, perioperative remifentanil and propofol doses, and length of surgical incision.

The primary outcome was pain score at rest during the first $48 \mathrm{~h}$ postoperative period using the VAS score (0 is defined as no pain and 10 as the maximum pain). The following variables were defined as secondary outcomes: sedation Ramsay scores, times of rescue analgesia requests, incidence of postoperative nausea and vomiting, antiemetic therapy requirements and incidence of pruritus (patients were asked about the desire to scratch). These variables were assessed at 2, 4, 6, 12, 24, 36 and 48 h postoperatively through a standardized questionnaire. Wound drainage at the catheter insertion site was assessed at 24 and $48 \mathrm{~h}$ postoperatively. The catheters were removed with aseptic technique when the drainage tube was taken off at $48 \mathrm{~h}$ in all patients. The surgery area was disinfected and dressed good after removing drainage tube and catheters. The tip of the catheters was subjected to microbiological analysis. No patients had malfunction or by obstruction (bending, seals 
traces of blood) or fever. In case of symptoms of CNS toxicity, such as numbness of the tongue, dizziness, visual disturbances, metallic taste, tinnitus, muscular twitching and dysarthria, or hemodynamic changes, the ropivacaine infusion was immediately discontinued.

Patients were discharged when they fulfilled the following criteria: normal body temperature, absence of anemia and hemodynamic impairment, absence of nausea and vomiting, VAS $\leq 3$ and independent ambulation. These criteria were assessed every day after surgery.

Three months after operation, patients were interviewed by an investigator. Patients were asked to answer yes/no to the following questions: residual pain (pain, pain to nonpainful stimulus, analgesic requirements) and surgical wound infection (and need for antibiotic therapy). Satisfaction with analgesic technique was evaluated by a 10-point scale $\quad(0=$ not $\quad$ satisfied, $10=$ maximum satisfaction).

\section{Statistical analysis}

Statistical analysis was performed using SPSS 16.0. Sample size calculation was based on the VAS $4 \mathrm{~h}$ after surgery in the pilot study $\left(\operatorname{mean}_{\mathrm{V}}=2.4, \mathrm{SD}_{\mathrm{V}}=0.7 ; \operatorname{mean}_{\mathrm{L}}=2.3\right.$, $\left.\mathrm{SD}_{\mathrm{L}}=0.5\right)$, with margin $=0.4, \quad 1-\beta=0.80$ and $\alpha=0.05$, therefore a sample size of 20 patients in each group was needed for testing non-inferiority. For the postoperative nausea and vomiting incidence outcome $\left(P_{\mathrm{V}}=20 \%\right.$ and $P_{\mathrm{L}}=0 \%$ in pilot trial), with the same $\alpha$ and $\beta$ values, a sample size of 35 patients per group was obtained.

Normally distributed continuous variables (patient characteristics and surgical information) were compared using two-group Student t test, whereas Mann-Whitney test with Bonferroni correction was used for comparison of VAS and Ramsay scores. The gender between two groups was compared using the $\chi^{2}$ test. The incidence of postoperative nausea and vomiting, pruritus, and fulfillment of discharge criteria werer analyzed with the Fisher exact test. Data collected at the 3-month follow-up call were analyzed with the Mann-Whitney $U$ test and the Fisher exact test. A $P<0.05$ was considered to be statistically significant in all cases except when the Bonferroni correction was performed for pain and sedation, in which case a $P<0.0071$ was considered significant (this value represents 0.05 divided by the number of repeated measures: 7 ).

\section{Results}

Overall, 94 patients were included in the study, and 23 patients were excluded after allocation (Fig. 1). All enrolled patients successfully completed the study and were included in the main analysis. Demographic data and surgical characteristics were not significantly different between the two groups (Table 1). No statistically significant differences were observed between the two groups with regard to operating time $(\mathrm{L}$ vs $\mathrm{V}, 251.91 \pm 82.58$ vs $246.39 \pm 84.72 \mathrm{~min} ; P=0.95)$.

Postoperative VAS scores at rest were not significantly different between two groups (Fig. 2). During the postoperative 2 days, rescue analgesic medications were required 14 times in the L group and 15 times in the control group. All wound catheters were removed at $48 \mathrm{~h}$ postoperatively without any complications, but microbiologic culture of catheter tip from one patient detected Serratia marcescens.

Several secondary outcomes, except for the incidence of postoperative nausea or vomiting $(P=0.025)$, did not show any significant difference between groups (Fig. 3; Table 2). Six patients in the $\mathrm{V}$ group experienced mild postoperative nausea or vomiting and this did not require discontinuation of the pump. No patients in the L group reported postoperative nausea or vomiting, or obvious CNS toxic signs of ropivacaine. No major adverse event occurred in any patient. Pruritus, dizziness and wound drainage in each group, and CNS symptoms of ropivacaine intoxication in $\mathrm{L}$ group were not found.

The length of hospital stay and comprehensive evaluation were not significantly different between the two groups (Table 3). All patients received the same pattern of analgesic treatment at hospital discharge and compliance rate. At 3 months after the surgery, 3 patients $(8.6 \%)$ in $\mathrm{L}$ group and 14 patients $(38.9 \%)$ in $\mathrm{V}$ group reported residual wound pain $(P<0.05)$. One patient in L group, whose microbiologic culture detected $S$. marcescens, returned to hospital for further treatment.

\section{Discussion}

Our research revealed that pain scores at rest during the $48 \mathrm{~h}$ postoperative period were not significantly different between the continuous ropivacaine wound infusion group and the ICCA group, suggesting that the two methods could achieve the same analgesic effect. More importantly, compared with the ICCA group, a positive effect on lightening chronic pain 3 months later and reducing the incidence of postoperative nausea and vomiting was existing.

In the research, we chose ropivacaine because of its lower systemic toxicity and its shorter elimination half-life, reducing the risk of plasma accumulation during prolonged infusion [6]. We used in this experiment $6 \mathrm{ml} 1 \%$ ropivacaine and followed by continuous infusion with $0.33 \%$ ropivacaine via a double lumen catheter system at a rate of $5 \mathrm{ml} / \mathrm{h}$ for $48 \mathrm{~h}$. This does/volume chosen was base on the reference [6]. 
Fig. 1 Flow diagram of the different trial phases

\section{Potential participants $(n=94)$}

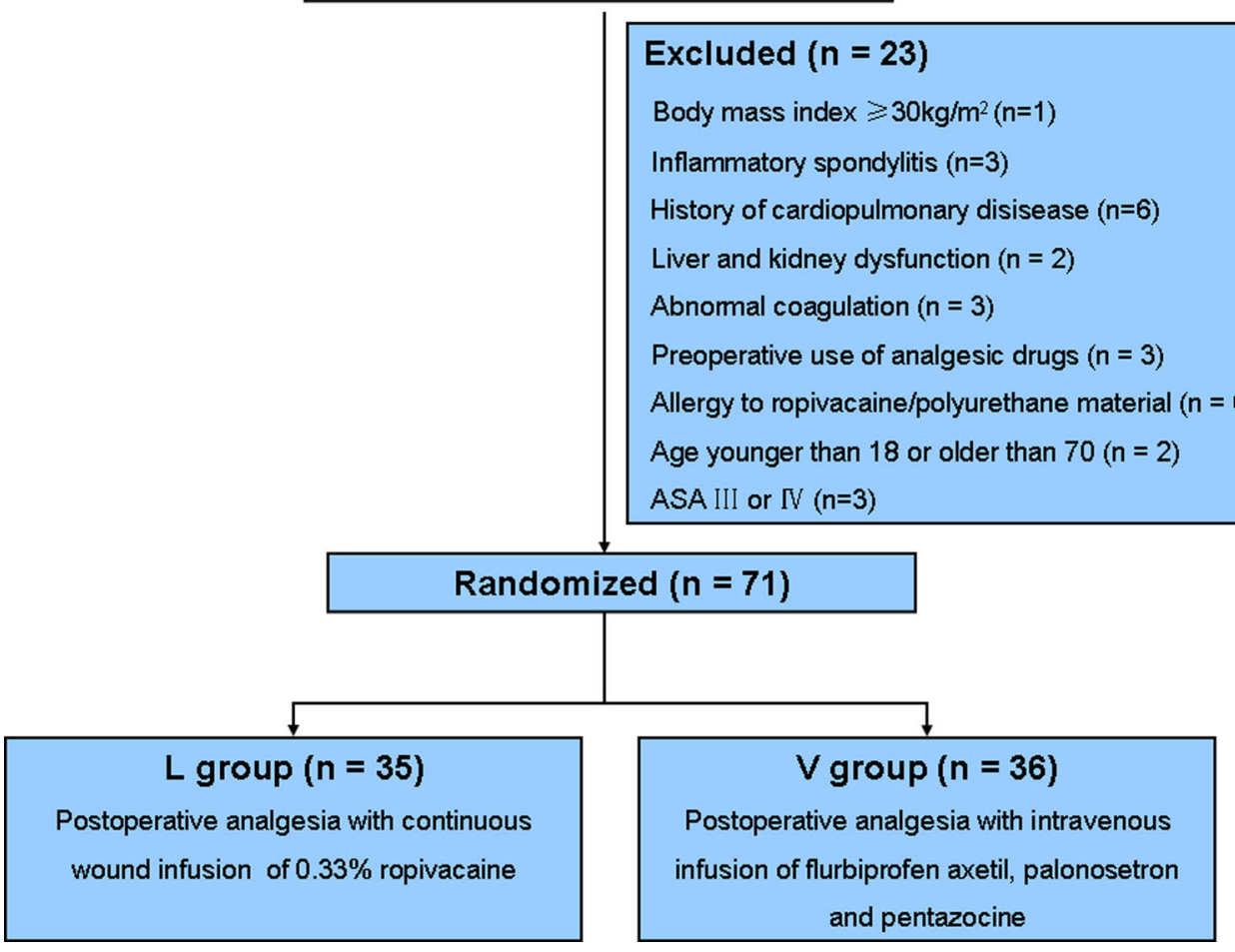

Table 1 Demographic data of the patients enrolled in the study

\begin{tabular}{|c|c|c|c|}
\hline & $\mathrm{L}$ group $(n=35)$ & $\mathrm{V}$ group $(n=36)$ & Significance \\
\hline Age (years) & $51.91 \pm 11.44$ & $49.06 \pm 11.20$ & ns \\
\hline Sex (male/female) & $19 / 16$ & $19 / 17$ & ns \\
\hline \multicolumn{4}{|l|}{ Disease } \\
\hline Lumbar fractures & 8 & 6 & \\
\hline Lumbar disc herniation and/or lumbar spondylolisthesis & 26 & 27 & \\
\hline Thoracic fractures & 1 & 2 & \\
\hline Thoracic disc herniation & 0 & 1 & \\
\hline Preoperative VAS & $1.51 \pm 0.66$ & $1.64 \pm 0.76$ & ns \\
\hline Operating time (min) & $251.91 \pm 82.58$ & $246.39 \pm 84.72$ & ns \\
\hline Length of surgical incision $(\mathrm{cm})$ & $10.37 \pm 4.39$ & $9.03 \pm 3.53$ & ns \\
\hline Perioperative remifentanil dose $(\mathrm{mg})$ & $3.03 \pm 0.70$ & $3.07 \pm 1.01$ & ns \\
\hline Perioperative propofol dose (mg) & $2148.15 \pm 477.47$ & $2123.33 \pm 710.84$ & ns \\
\hline
\end{tabular}

Data shown as mean \pm SD (95\% confidence interval)

$n s$ not significant

The optimal method of providing analgesia with continuous wound infusion with local anesthetic in spinal surgery is still not conclusive. Variables such as the surgical plane in which the catheter is placed, the length and type of the catheter, the dose and duration of infusion still remain undetermined. Bianconi et al. [7] used continuous wound infusion with catheters placed between muscle fascia and subcutaneous tissue after spinal surgery. And they reported significant analgesic efficacy both in reducing pain scores and opioid use. However, studies have shown that subcutaneous infiltrations may not improve postoperative pain scores, although they do have an impact on opioid consumption [8,9]. When compared with subfascial injection, the deeper infiltration results in better efficacy [10]. Therefore, we chose the above and below the muscle fascia catheter location. In this study, none of our catheter implantations affected wound drainage. 


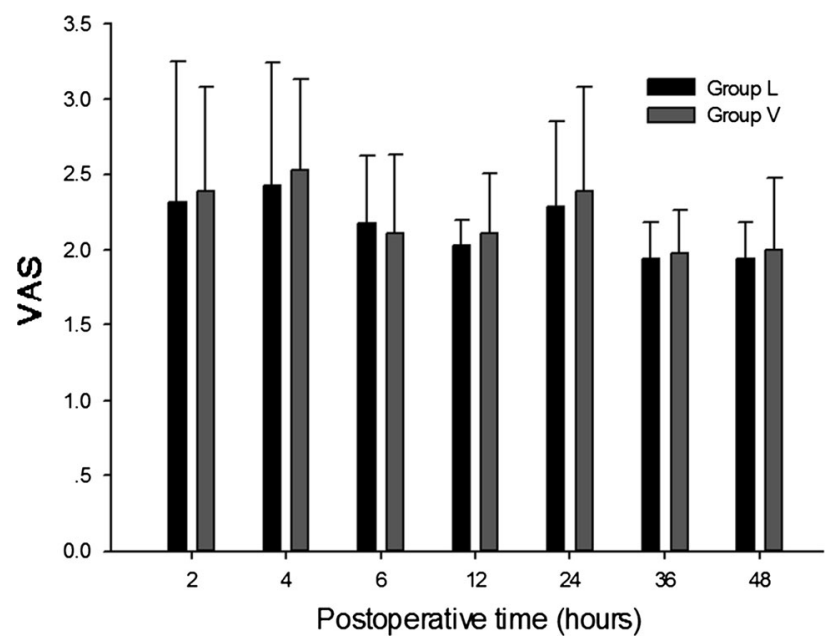

Fig. 2 VAS for pain at rest in the L group and V group at 2, 4, 6, 12, 24,36 and $48 \mathrm{~h}$ after surgery. Data are reported as mean $\pm \mathrm{SD}$

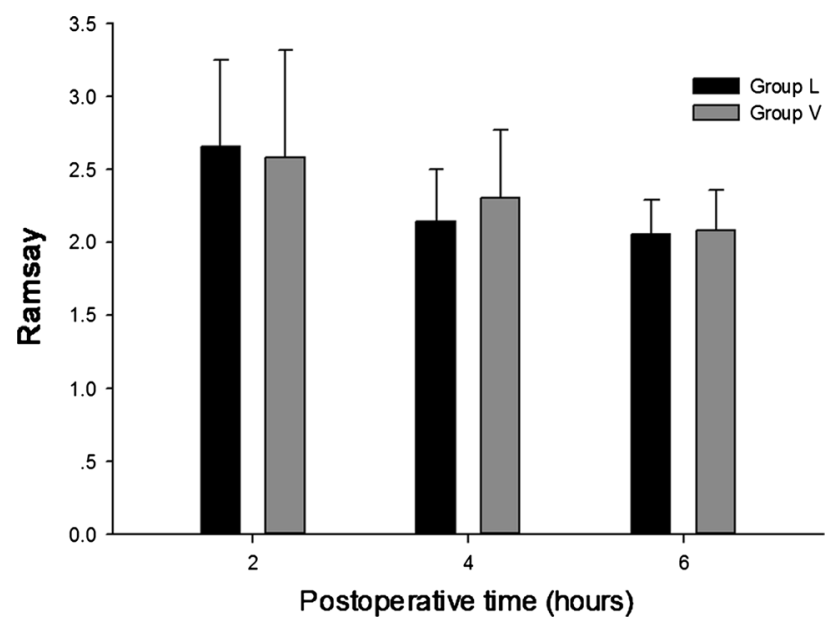

Fig. 3 Ramsay scores for sedation in the $\mathrm{L}$ group and $\mathrm{V}$ group at 2, 4 and $6 \mathrm{~h}$ after surgery. At 12, 36 and $48 \mathrm{~h}$, the Ramsay scores were 2. Data are reported as mean $\pm \mathrm{SD}$

Table 2 Postoperative side effects

\begin{tabular}{llll}
\hline & $\begin{array}{l}\text { L group } \\
(n=35)\end{array}$ & $\begin{array}{l}\text { V group } \\
(n=36)\end{array}$ & $P$ \\
\hline Nausea/vomiting & 0 & 6 & 0.012 \\
Pruritus & 0 & 0 & \\
Dizziness & 0 & 0 & \\
Wound exudation & 0 & 0 & \\
\hline
\end{tabular}

Analysis with Fisher exact test

Although some authors [11, 12] have concerns that wound infiltration with local anesthetic may interfere with wound closure and normal wound healing. Until now, there is no definitive data on mature wound strength after wound infusion analgesia. Hopf et al. [13] highlighted that postoperative pain could affect the inflammatory response and increase catecholamine release, leading to wound perfusion and oxygenation decline. Conversely, it is possible that partial blockade of the inflammatory response in the first phase of wound closure by wound infiltration with local anesthetics through providing good pain control may be beneficial and result in less fibroblast hyperplasia. As Watanabe et al. [14] suggests, fluid infused into a wound with saline may have an effect in decreasing the pain response because it can dilute and wash out pain substances such as histamine and vasoactive polypeptides.

Questions remain regarding the potential risk of wound infection when providing analgesia with continuous wound infusion with local anesthetic. Our microbiologic culture results showed $S$. marcescens in one patient in the $\mathrm{L}$ group. The reason was unknown. But since non-thorough aseptic procedure during routine surgeries also occasionally causes Serratia, we believe this case is not directly related to the catheter implantation. Moreover, the wound infection rates did not differ between the two groups $(P>0.05)$, suggesting that the catheter does not increase the risk of wound infection in local anesthetic infusion patients. In a metaanalysis by Liu et al. [4], the overall wound infection rate was similar between catheter with local anesthetic $(0.7 \%)$ and catheter with placebo or the no-catheter control group $(1.2 \%)$. It was also reported that the incidence of catheter or pump failure was $1.1 \%$. Although it has been reported that the local anesthetic ropivacaine displays antimicrobial activity [15], a study of continuous ropivacaine infusion in the median sternotomy incision following cardiac surgery showed high infection rates $(9 \%, n=44)$ and this rate of infection was not statistically different in the saline group $(0 \%, n=41)(P>0.05)$ [16]. However, a larger sample size must be evaluated before a definitive conclusion can be drawn about the safety of this technique.

Chronic pain is known to severely affect quality of life and has significant economic consequences [6]. On stripping of muscle attachment points and injury of soft tissue in our research, there was an 8.6 and $38.9 \%$ incidence of dull pain within the local operative area independent of the primary pain in $\mathrm{L}$ group and $\mathrm{V}$ group, respectively $(P<0.05)$. We suspect that the local anesthetic acts directly on the surgical site, cutting off the source of harmful stimulation, this might cause the difference between two groups. An recent experimental study on rat, mouse, monkey and humans has suggested that a long noncoding RNA contributes to neuropathic pain following nerve injury [17]. And Attal et al. [18] in a double-blind controlled crossover study with a 2-week washout period evaluated the effect of lidocaine. A decrease in spontaneous pain, mechanical dynamic allodynia, static mechanical allodynia, and hyperalgesia was demonstrated. It was thought that washout of the injured site was dedicated to mitigating to 
Table 3 Length of hospital stay and satisfaction

\begin{tabular}{llll}
\hline & $\begin{array}{l}\text { L group } \\
(n=35)\end{array}$ & $\begin{array}{l}\text { V group } \\
(n=36)\end{array}$ & Significance \\
\hline Length of hospital stay & $10.00 \pm 5.79$ & $8.94 \pm 3.85$ & $\mathrm{~ns}$ \\
Satisfaction & $8.80 \pm 0.58$ & $8.83 \pm 0.56$ & $\mathrm{~ns}$ \\
\hline
\end{tabular}

Analysis with independent samples $T$ test

$n s$ not significant

peripheral neuropathic pain. In recent pharmaceutical developments, flurbiprofen has been introduced as a parental drug carrier acting as a sustained-release formulation and targeting injured tissue [6]. However, flurbiprofen was mainly persisted retention in the blood circulation and passively targeted to the site of injury with a highly permeable capillary endothelium. The transmission of pain stimuli to the spinal cord cannot be inhibited completely by flurbiprofen, which leads to long noncoding RNA changes, and less effective inhibition of the incidence of chronic pain than the local anesthetics.

In conclusion, the efficacy of continuous surgical wound infusion with local anesthetics was tested using multiholed catheters placed above and below the muscle fascia after thoracolumbar spinal surgery. Our study showed that continuous wound analgesia infusion pump could achieve the same analgesic effect as ICCA during hospitalization after spine surgery, while avoiding intravenous analgesic drug-induced adverse effects such as postoperative nausea and vomiting. More importantly, continuous surgical wound infusion with local anesthetics is superior to ICCA in reducing the incidence of chronic pain. Since the number of patients in our study is small, the risk of bias cannot be ignored when we make such conclusion. However, this therapy could be an important component of multimodal approaches for postoperative pain management.

Conflict of interest All the authors declare they have no competing of interests.

Open Access This article is distributed under the terms of the Creative Commons Attribution 4.0 International License (http:// creativecommons.org/licenses/by/4.0/), which permits unrestricted use, distribution, and reproduction in any medium, provided you give appropriate credit to the original author(s) and the source, provide a link to the Creative Commons license, and indicate if changes were made.

\section{References}

1. DeVine J, Norvell DC, Ecker E, Fourney DR, Vaccaro A, Wang J, Andersson G (2011) Evaluating the correlation and responsiveness of patient-reported pain with function and quality-of-life outcomes after spine surgery. Spine (Phila Pa 1976) 36(21 Suppl):S69-S74. doi:10.1097/BRS.0b013e31822ef6de
2. Mathiesen O, Dahl B, Thomsen BA, Kitter B, Sonne N, Dahl JB, Kehlet H (2013) A comprehensive multimodal pain treatment reduces opioid consumption after multilevel spine surgery. Eur Spine J 22(9):2089-2096. doi:10.1007/s00586-013-2826-1

3. Forastiere E, Sofra M, Giannarelli D, Fabrizi L, Simone G (2008) Effectiveness of continuous wound infusion of $0.5 \%$ ropivacaine by On-Q pain relief system for postoperative pain management after open nephrectomy. Br J Anaesth 101(6):841-847. doi:10. 1093/bja/aen309

4. Liu SS, Richman JM, Thirlby RC, Wu CL (2006) Efficacy of continuous wound catheters delivering local anesthetic for postoperative analgesia: a quantitative and qualitative systematic review of randomized controlled trials. J Am Coll Surg 203(6):914-932. doi:10.1016/j.jamcollsurg.2006.08.007

5. Rushdi T, Kholeif A, Ibrahim M (2011) Ropivacaine 0.4\% iontophoresis versus continuous catheter infusion after iliac crest bone grafting in lumbar spine surgery. Egypt $\mathrm{J}$ Anaesth 27:261-266

6. McCarberg BH, Nicholson BD, Todd KH, Palmer T, Penles L (2008) The impact of pain on quality of life and the unmet needs of pain management: results from pain sufferers and physicians participating in an internet survey. Am J Ther 15(4):312-320. doi:10.1097/MJT.0b013e31818164f2

7. Bianconi M, Ferraro L, Ricci R, Zanoli G, Antonelli T, Giulia B, Guberti A, Massari L (2004) The pharmacokinetics and efficacy of ropivacaine continuous wound instillation after spine fusion surgery. Anesth Analg 98(1):166-172 (Table of contents)

8. Brown TD (2013) Spinal cord stimulation for chronic pain. In: Minimally Invasive Spine Surgery, Chap. 27. pp 251-259

9. Kainu JP, Sarvela J, Halonen P, Puro H, Toivonen HJ, Halmesmaki E, Korttila KT (2012) Continuous wound infusion with ropivacaine fails to provide adequate analgesia after caesarean section. Int J Obstet Anesth 21(2):119-124. doi:10.1016/j.ijoa. 2011.12.009

10. Kong TW, Park H, Cheong JY, Min SK, Ryu HS (2014) Efficacy of continuous wound infiltration of local anesthetic for pain relief after gynecologic laparoscopy. Int $J$ Gynaecol Obstet 124(3):212-215. doi:10.1016/j.ijgo.2013.08.019

11. Lucchinetti E, Awad AE, Rahman M, Feng J, Lou PH, Zhang L, Ionescu L, Lemieux H, Thebaud B, Zaugg M (2012) Antiproliferative effects of local anesthetics on mesenchymal stem cells: potential implications for tumor spreading and wound healing. Anesthesiology 116(4):841-856. doi:10.1097/ALN. 0b013e31824babfe

12. Baxter R, Bramlett K, Onel E, Daniels S (2013) Impact of local administration of liposome bupivacaine for postsurgical analgesia on wound healing: a review of data from ten prospective, controlled clinical studies. Clin Ther 35(3):312-320 e315. doi:10. 1016/j.clinthera.2013.02.005

13. Hopf HW, Hunt TK, West JM, Blomquist P, Goodson WH 3rd, Jensen JA, Jonsson K, Paty PB, Rabkin JM, Upton RA, von Smitten K, Whitney JD (1997) Wound tissue oxygen tension predicts the risk of wound infection in surgical patients. Arch Surg 132(9):997-1004 (Discussion 1005)

14. Watanabe M, Sakai D, Matsuyama D, Yamamoto Y, Sato M, Mochida J (2010) Risk factors for surgical site infection following spine surgery: efficacy of intraoperative saline irrigation. J Neurosurg Spine 12(5):540-546. doi:10.3171/2009.11. SPINE09308

15. Johnson SM, Saint John BE, Dine AP (2008) Local anesthetics as antimicrobial agents: a review. Surg Infect (Larchmt) 9(2):205-213. doi:10.1089/sur.2007.036

16. Agarwal S, Nuttall GA, Johnson ME, Hanson AC, Oliver WC Jr (2013) A prospective, randomized, blinded study of continuous ropivacaine infusion in the median sternotomy incision following 
cardiac surgery. Reg Anesth Pain Med 38(2):145-150. doi:10. 1097/AAP.0b013e318281a348

17. Zhao X, Tang Z, Zhang H, Atianjoh FE, Zhao JY, Liang L, Wang W, Guan X, Kao SC, Tiwari V, Gao YJ, Hoffman PN, Cui H, Li M, Dong X, Tao YX (2013) A long noncoding RNA contributes to neuropathic pain by silencing Kcna2 in primary afferent neurons. Nat Neurosci 16(8):1024-1031. doi:10.1038/nn.3438

18. Attal N, Rouaud J, Brasseur L, Chauvin M, Bouhassira D (2004) Systemic lidocaine in pain due to peripheral nerve injury and predictors of response. Neurology 62(2):218-225 